\title{
WEIGHT SPACES AND IRREDUCIBLE REPRESENTATIONS OF SIMPLE LIE ALGEBRAS
}

F. W. LEMIRE

Let $L$ denote a simple Lie algebra over an algebraically closed field $K$ of characteristic zero. Harish-Chandra [2] has shown that to each one-dimensional representation $(\lambda, K)$ of the Cartan subalgebra TC of $L$ we may associate an irreducible representation of $L$ admitting $\lambda$ as a "highest weight function". This has been generalized by the author [3] by showing that if $\mathcal{C}$ is the centralizer of $\mathfrak{H}$ in $U$, the universal enveloping algebra of $L$, and $(\gamma, K)$ is a one-dimensional representation of $\mathfrak{C}$ we may again construct an irreducible representation of $L$ admitting $\gamma \downarrow \mathcal{H}$ as a weight function. In this paper we make a further study of the relationship between the representations of $L$ and their weight spaces.

1. Weight spaces of irreducible representations. It is well known that there exists a one-one correspondence between the representations of $L$ and those of $U$ which preserves irreducibility. Throughout this paper we will not distinguish between a representation of $L$ and its unique extension to $U$.

Definition 1. Let $(\rho, V)$ be a representation of $L$. Then for each linear functional $\lambda \in \mathcal{H}^{*}$, the dual linear space of the Cartan subalgebra, we define

$$
V_{\lambda}=\{v \in V \mid \rho(H) v=\lambda(H) v(\forall H \in \mathfrak{C})\} .
$$

Also we denote by $[\rho: \lambda]$ the dimension of the subspace $V_{\lambda}$. If, in particular, $[\rho: \lambda]>0$ we call $\lambda$ a weight function of the representation $\rho$ and $V_{\lambda}$ the corresponding weight space.

It is clear from the definitions of $\mathcal{C}$ and $V_{\lambda}$ that for any element $c \in \mathcal{C}, \rho(c)$ maps $V_{\lambda}$ into $V_{\lambda}$.

Definition 2. Let $(\rho, V)$ be a representation of $L$ and $\lambda \in \mathcal{H}^{*}$. Then we define a representation $\left(\eta(\rho, \lambda), V_{\lambda}\right)$ of $\mathfrak{e}$ by setting $\eta(\rho, \lambda)(c)$ $=\rho(c) \downarrow V_{\lambda}$ for all $c \in \mathcal{C}$.

LEMMA 1. If $(\rho, V)$ is an irreducible representation of $L$ and $\lambda \in \mathcal{H}^{*}$ such that $[\rho: \lambda]>0$ then $\left(\eta(\rho, \lambda), V_{\lambda}\right)$ is a nontrivial, irreducible representation of $\mathrm{C}$.

Presented to the Society, August 28, 1968 under the title Weight spaces and irreducible representations; received by the editors September 10, 1968. 
Proof. It suffices to show that for an arbitrary nonzero element $v \in V_{\lambda}$ we have $\eta(\rho, \lambda)(\mathcal{e})(v)=V_{\lambda}$, and this follows as a special case of equation (2.4) in a paper by Bouwer [1].

We now observe that the representation $\eta(\rho, \lambda)$ determines $\rho$ in the following sense.

LEMMA 2. Given two irreducible representations $\left(\rho_{1}, V_{1}\right)$ and $\left(\rho_{2}, V_{2}\right)$ of $L$ such that there exists a linear functional $\lambda \in \mathfrak{H}^{*}$ with $\left[\rho_{1}: \lambda\right]$ and $\left[\rho_{2}: \lambda\right]>0$ and $\eta\left(\rho_{1}, \lambda\right)$ equivalent to $\eta\left(\rho_{2}, \lambda\right)$, then $\rho_{1}$ is equivalent to $\rho_{2}$.

Proof. We first observe that if $M$ is a maximal left ideal of $\mathcal{C}$ then there exists a unique maximal left ideal $M^{\prime}$ of $U$ containing $M$. In fact, if $M^{\prime \prime}$ denotes the left ideal of $U$ generated by $M$ we can easily see that $M^{\prime \prime} \neq U$ and hence the existence of $M^{\prime}$ is established by a simple application of Zorn's Lemma. For the uniqueness it suffices to show that the left regular representation $\left(\pi, U / M^{\prime \prime}\right)$ of $U$ modulo $M^{\prime \prime}$ has a unique maximal $\pi(U)$-invariant subspace. Clearly $U / M^{\prime \prime}$ $=\sum \oplus\left(U / M^{\prime \prime}\right)_{\gamma}$ and if $W$ is a $\pi(U)$-invariant subspace of $U / M^{\prime \prime}$ we have $W=\sum \oplus\left(W \cap\left(U / M^{\prime \prime}\right)_{\gamma}\right)$. Since $\left(U / M^{\prime \prime}\right)_{\lambda}$ is the representation space of an irreducible representation of $\mathfrak{e}$ it follows that $W \cap$ $\left(U / M^{\prime \prime}\right)_{\lambda}=\left(U / M^{\prime \prime}\right)_{\lambda}$ or $W \cap\left(U / M^{\prime \prime}\right)_{\lambda}=\{0\}$. In the first case $1+M^{\prime \prime} \in W$ and hence $W=U / M^{\prime \prime}$. Therefore every proper $\pi(U)$ invariant subspace of $U / M^{\prime \prime}$ is a subspace of $\sum_{\gamma \neq \lambda} \oplus\left(U / M^{\prime \prime}\right)_{\gamma}$ $\neq U / M^{\prime \prime}$, and hence $W_{s}$, the sum of all proper $\pi(U)$-invariant subspaces of $U / M^{\prime \prime}$, is the unique maximal proper $\pi(U)$-invariant subspace of $U / M^{\prime \prime}$.

Next suppose $M_{1}$ and $M_{2}$ are two maximal left ideals of $\mathfrak{e}$ such that the left regular representations of e modulo $M_{1}$ and $\mathfrak{e}$ modulo $M_{2}$ are equivalent. Then we claim that the left regular representations of $U$ modulo $M_{1}^{\prime}$ and $U$ modulo $M_{2}^{\prime}$ are equivalent. Indeed by assumption we have a $\mathcal{C}$-linear map $\phi: \mathbb{e} / M_{1} \rightarrow \mathfrak{e} / M_{2}$. Let $\phi\left(1+M_{1}\right)=x+M_{2}$ and define $\phi^{\prime}: U / M_{1}^{\prime} \rightarrow U / M_{2}^{\prime}$ by setting $\phi^{\prime}\left(u+M_{1}^{\prime}\right)=u x+M_{2}^{\prime}$. It is then readily checked that $\phi^{\prime}$ establishes the required equivalence.

Finally assume $\left(\rho_{1}, V_{1}\right)$ and $\left(\rho_{2}, V_{2}\right)$ are two irreducible representations of $L$ such that there exists a linear functional $\lambda \in \mathcal{F}^{*}$ with $\left[\rho_{i}: \lambda\right]>0$ and the representations $\left(\eta\left(\rho_{1}, \lambda\right),\left(V_{1}\right)_{\lambda}\right)$ and $\left(\eta\left(\rho_{2}, \lambda\right),\left(V_{2}\right)_{\lambda}\right)$ are equivalent. Choose nonzero elements $v_{i} \in\left(V_{i}\right)_{\lambda}$ and define $M_{i}=\left\{c \in \mathfrak{e} \mid \eta\left(\rho_{i}, \lambda\right)(c) v_{i}=0\right\}$ for $i=1$, 2. Clearly $M_{i}$ is a maximal left ideal in $\mathcal{C}$ and $\left(\eta\left(\rho_{i}, \lambda\right),\left(V_{i}\right)_{\lambda}\right)$ is equivalent to the left regular representation of $\mathfrak{e}$ modulo $M_{i}$. By assumption then the left regular representations of $\mathcal{e}$ modulo $M_{1}$ and $\mathfrak{e}$ modulo $M_{2}$ are equivalent. As above, this implies that the left regular representations of $U$ modulo $M_{1}^{\prime}$ and $U$ modulo $M_{2}^{\prime}$ are equivalent. Then, by the unique- 
ness of $M_{i}^{\prime}$ we have $M_{i}^{\prime}=\left\{u \in U \mid \rho_{i}(u) v_{i}=0\right\}$ and $\left(\rho_{i}, V_{i}\right)$ is equivalent to the left regular representation of $U$ modulo $M_{i}^{\prime}$. Therefore $\left(\rho_{1}, V_{1}\right)$ is equivalent to $\left(\rho_{2}, V_{2}\right)$.

The result of Lemma 2 suggests a closer look at the irreducible representations of $\mathfrak{C}$.

Definition 3. A nontrivial representation $(\eta, W)$ of $\mathfrak{e}$ is said to be $\lambda$-weighted for some linear functional $\lambda \in \mathcal{F}^{*}$ iff it is irreducible and $\eta(H-\lambda(H) \cdot 1)=0$ for all $H \in \mathcal{H}$.

Lemma 3. Every $\lambda$-weighted representation of $\mathfrak{C}$ is equivalent to a representation of the form $\left(\eta(\rho, \lambda), V_{\lambda}\right)$ for some irreducible representation $(\rho, V)$ of $L$ with $[\rho: \lambda]>0$.

Proof. Let $(\eta, W)$ be a $\lambda$-weighted representation of $\mathfrak{e}$ and select a nonzero element $w \in W$. Set $M=\{c \in \mathfrak{C} \mid \eta(c) w=0\}$. Clearly $M$ is a maximal left ideal of $\mathfrak{C}$. Now we claim that the left regular representation of $U$ modulo $M^{\prime}$ (the unique maximal left ideal of $U$ containing $M$ ) is the required irreducible representation. This follows immediately on observing that $M^{\prime} \cap \mathfrak{e}=M$. To prove this latter fact we note that $M \subseteq M^{\prime} \cap \mathfrak{e}$ by definition of $M^{\prime}$. Then take any $x \in M^{\prime} \cap \mathfrak{e}$ and assume $x \in M$. By the maximality of $M$ in $\mathfrak{e}$ there exists an element $y \in \mathbb{C}$ such that $y x-1 \in M$ and hence $1 \in M^{\prime}$. This contradiction implies that $M=M^{\prime} \cap \mathfrak{e}$.

Finally combining the results of this section we have

Theorem 1. For any fixed linear functional $\lambda \in \mathcal{H C}^{*}$ there is a one-toone correspondence between the set of equivalence classes of irreducible representations $(\rho, V)$ of $L$ with $[\rho: \lambda]>0$ and the set of equivalence classes of $\lambda$-weighted representations of $\mathrm{e}$.

2. Relations between weight spaces. Two natural questions now arise:

(1) Suppose $(\rho, V)$ is an irreducible representation of $L$ such that $\left[\rho: \lambda_{1}\right]>0$ and $\left[\rho: \lambda_{2}\right]>0$ for two different linear functionals $\lambda_{1}$, $\lambda_{2} \in \mathfrak{H}^{*}$. Then how are the representations $\eta\left(\rho, \lambda_{1}\right)$ and $\eta\left(\rho, \lambda_{2}\right)$ related?

(2) Conversely, let $\left(\eta_{i}, W_{i}\right)$ be a $\lambda_{i}$-weighted representation of $\mathfrak{e}$ for $i=1$, 2. If $\lambda_{1} \neq \lambda_{2}$, under what conditions does there exist a common irreducible representation $(\rho, V)$ of $L$ such that $\eta_{i}$ is equivalent to $\eta\left(\rho, \lambda_{i}\right)$ for $i=1,2$ ?

Unfortunately we have been unable to provide a "e-internal" answer to these questions; however, we do have the following straightforward result which we will use in the next section for somewhat more satisfactory results in a restricted case. 
TheOREM 2. Let $\left(\eta_{i}, W_{i}\right)$ be a $\lambda_{i}$-weighted representation of $\mathfrak{e}$ and let $M_{i}$ denote a maximal left ideal of $\mathfrak{C}$ such that $\eta_{i}$ is equivalent to the left regular representation of $\mathfrak{C}$ modulo $M_{i}$ for $i=1,2$. Then there exists an irreducible representation $(\rho, V)$ of $L$ such that $\eta_{i}$ is equivalent to $\eta\left(\rho, \lambda_{i}\right)(i=1,2)$ iff there exists an element $x \in U-M_{2}^{\prime}$ such that $M_{1}^{\prime} x \subseteq M_{2}^{\prime}$.

Proof. If there exists an element $x \in U-M_{2}^{\prime}$ such that $M_{1}^{\prime} x \subseteq M_{2}^{\prime}$ then the map $\phi: U / M_{1}^{\prime} \rightarrow U / M_{2}^{\prime}$ defined by $\phi\left(u+M_{1}^{\prime}\right)=u x+M_{2}^{\prime}$ is a linear isomorphism which establishes the equivalence between the left regular representation of $U$ modulo $M_{1}^{\prime}$ and of $U$ modulo $M_{2}^{\prime}$. Let $(\rho, V)$ be any representation in this equivalence class; it is clear that $\eta_{i}$ is equivalent to $\eta\left(\rho, \lambda_{i}\right)$ of $i=1,2$.

Conversely if there exists an irreducible representation $(\rho, V)$ of $L$ such that $\eta_{i}$ is equivalent to $\eta\left(\rho, \lambda_{i}\right)$ then $M_{i}$ can be considered to be the left annihilator ideal of some nonzero element $v_{i} \in V_{\lambda_{i}}$-i.e. $M_{i}=\left\{c \in \mathcal{C} \mid \rho(c) v_{i}=0\right\}$-for $i=1$, 2. Moreover, as in $\$ 1$ we have $M_{i}^{\prime}=\left\{u \in U \mid \rho(u) v_{i}=0\right\}$ for $i=1,2$. Since $\rho$ is assumped to be irreducible there exists an element $x \in U$ such that $\rho(x) v_{2}=v_{1}$ and hence $x \notin M_{2}^{\prime}$ and $M_{1}^{\prime} x \subseteq M_{2}^{\prime}$.

3. One-dimensional weight spaces. In the case of all finite-dimensional irreducible representations or, more generally, all irreducible representations $(\rho, V)$ of $L$ admitting a "highest weight", it is well known that there always exists at least one linear functional $\lambda \in \mathfrak{H}^{*}$ such that $[\rho: \lambda]=1$. On the other hand, if $(\rho, V)$ is an irreducible representation of $L$ for which there exists a linear functional $\lambda \in \mathcal{F}^{*}$ with $[\rho: \lambda]=1$ then, as above, $\rho$ is determined by $\eta(\rho, \lambda)$ and moreover $\eta(\rho, \lambda)$ may be regarded as an algebra homomorphism from $\mathcal{e}$ into $K$. This particular class of representations of $L$ was studied by the author in a previous paper [3]. Unfortunately, it is possible for inequivalent one-dimensional representations of $\mathfrak{C}$ to yield equivalent representations of $L$. The next two theorems are aimed at shedding some light on the relationship between one-dimensional representations of $\mathfrak{e}$ which yield equivalent representations of $L$.

TheOREm 3. Let $\left(\eta_{i}, K\right)$ be a $\lambda_{i}$-weighted one-dimensional representation of $\mathfrak{C}$ for $i=1,2$. If there exists an irreducible representation $(\rho, V)$ of $L$ such that $\eta_{i}$ is equivalent to $\eta\left(\rho, \lambda_{i}\right)$ for $i=1,2$ then there exist elements $x, y \in U$ such that $y x \in \mathcal{C}, \eta_{2}(y x)=1$ and $\eta_{1}(c)=\eta_{2}(y c x)$ for all $c \in \mathbb{e}$. 
Proof. Choose $0 \neq v_{i} \in V_{\lambda_{i}}$. Then $M_{i}=\left\{c \in \mathcal{C} \mid \rho(c) v_{i}=0\right\}$ is a maximal leftideal of $\mathcal{C}$ such that $\eta_{i}$ is equivalent to the left regular representation of $\mathfrak{C}$ modulo $M_{i}$. By Theorem 2 there exists an element $x \in U$ $-M_{2}^{\prime}$ such that $M_{1}^{\prime} x \subseteq M_{2}^{\prime}$. Since $x \notin M_{2}^{\prime}$ there exists an element $y \in U$ such that $y x-1 \in M_{2}^{\prime}$. Then clearly $y x \in \mathcal{C}$ and $\eta_{2}(y x)=1$. Finally, since $\eta_{2}\left(y\left(c-\eta_{1}(c) \cdot 1\right) x\right)=0, \eta_{1}(c)=\eta_{2}(y c x)$ for all $c \in \mathbb{e}$.

As a partial converse to Theorem 3 we have:

TheOREM 4. Let $\left(\eta_{i}, K\right)$ be a one-dimensional $\lambda_{i}$-weighted representation of $\mathcal{C}$ and denote $\operatorname{Ker}\left(\eta_{i}\right)$ by $M_{i}$ for $i=1,2$. If there exists an element $x \in U-M_{2}^{\prime}$ such that for all $y \in U$ satisfying $y x \in \mathbb{C}$ we have $\eta_{2}(y c x)$ $=\eta_{1}(c) \eta_{2}(y x)$ for all $c \in \mathcal{C}$ then there exists an irreducible representation $(\rho, V)$ of $L$ such that $\eta_{i}$ is equivalent to $\eta\left(\rho, \lambda_{i}\right)$ for $i=1,2$.

Proof. We first observe that the set

$$
\left\{u \in U \mid(\forall y \in U: y u \in \mathcal{C}) y u \in M_{i}\right\}
$$

is a maximal left ideal of $U$ containing $M_{i}$ and hence is equal to $M_{i}^{\prime}$.

Let $\left(\pi, U / M_{2}^{\prime}\right)$ denote the left regular representation of $U$ modulo $M_{2}^{\prime}$. For all $c \in \mathcal{C}$ we have $c x \equiv \eta_{1}(c) x \bmod M_{2}^{\prime}$. Suppose, to the contrary, that $c x-\eta_{1}(c) x \notin M_{2}^{\prime}$. By maximality of $M_{2}^{\prime}$, there exists an element $y \in U$ such that $y\left(c x-\eta_{1}(c) x\right)-1 \in M_{2}^{\prime}$-i.e. $\eta_{2}\left(y c x-\eta_{1}(c) y x\right)$ $=1$. However, by assumption $\eta_{2}\left(y c x-\eta_{1}(c) y x\right)=0$. This contradiction implies that $c x-\eta_{1}(c) x \in M_{2}^{\prime}$. Therefore $\eta_{1}$ is equivalent to $\eta\left(\pi, \lambda_{1}\right)$.

4. Some interesting questions. Having established the close relationship between the representations of $L$ and those of $\mathfrak{C}$, we are now interested in looking at the irreducible representations of $\mathfrak{C}$ and the structure of $\mathfrak{C}$. In this regard we have far more questions than answers. In previous papers [3], [4] we have shown that $\mathcal{C}$ is a finitely generated subalgebra of $U$ and if $(\eta, W)$ is a finite-dimensional, $\lambda$ weighted representation of $\mathfrak{C}$ then the associated irreducible representation $(\rho, V)$ of $L$ has the property that $[\rho: \lambda]<\infty$ for all $\lambda \in \mathcal{H}^{*}$.

From these observed facts we face the following questions:

(1) Are all $\lambda$-weighted (resp. irreducible) representations of $\mathfrak{C}$ finite-dimensional?

(2) Are all irreducible representations of $\mathfrak{e} \lambda$-weighted for some linear functional $\lambda \in \mathcal{H}^{*}$ ?

(3) If $(\eta, W)$ is a finite-dimensional $\lambda_{0}$-weighted representation of $L$, does there exist a linear functional $\lambda \in \mathcal{F}^{*}$ such that $[\rho: \lambda]=1$ ? 


\section{BIBLIOGRAPHY}

1. I. Z. Bouwer, Standard representations of simple Lie algebras, Canad. J. Math. 20 (1968), 344-361.

2. Harish-Chandra, On some applications of the universal enveloping algebra of a semisimple Lie algebra, Trans. Amer. Math. Soc. 70 (1951), 28-96.

3. F. W. Lemire, Irreducible representations of a simple Lie algebra admitting a one-dimensional weight space, Proc. Amer. Math. Soc. 19 (1968), 1161-1164.

4. - Note on weight spaces of irreducible linear representations, Canad. Math. Bull. 11 (1968), 399-403.

University of British Columbia 